International Mathematical Forum, 2, 2007, no. 50, 2453 - 2462

\title{
Existence Results for Generalized Vector Variational-like Inequalities ${ }^{1}$
}

\author{
Yali Zhao $^{a}{ }^{2}$, Chunguang Wang $^{b}$, Weiyi $\operatorname{Qian}^{a}$ and Zunquan Xia ${ }^{c}$ \\ ${ }^{a}$ Department of Mathematics, Bohai University \\ Jinzhou, Liaoning 121000, P. R. China \\ ${ }^{b}$ Department of Mathematics and Computer Science \\ Chaoyang Teachers College \\ Chaoyang, Liaoning 122000, P. R. China \\ ${ }^{c}$ Department of Applied Mathematics \\ Dalian University of Technology \\ Dalian, Liaoning 116024, P. R. China
}

\begin{abstract}
In this paper, we consider a vector version of Minty's Lemma and obtain existence theorems of solutions for two kinds of vector variationallike inequalities.
\end{abstract}

Mathematics Subject Classification: 49J40, 90C29

Keywords: Generalized vector variational-like inequality, Minty's lemma, KKM-mapping, Affine mapping, Cone

\section{Introduction}

A vector variational inequality (for short, VVI) in a finite-dimensional Euclidean space was first introduced by Giannessi [1]. This is a generalization of a scalar variational inequality to the vector case by virtue of multi-criterion consideration. Later on, many authors have investigated vector variational inequalities in abstract spaces, see $[2,3,6,7,9,13,14,19]$ and the references therein.

On the other hand, Minty's lemma $[5,10]$ has been shown to be an important tool in the variational field including variational inequality problems,

\footnotetext{
${ }^{1}$ This work is supported by the National Natural Science Foundation of China(10471015).

${ }^{2}$ Corresponding author, e-mail: yalizhao2000@yahoo.com.cn
} 
obstacle problems, confined plasmas, free boundary problems, and stochastic optimal control problems when the operator is monotone and the domain is convex.

In 1999, Lee at al. [16] obtained a vector version of Minty's lemma using Nadler's result [17], and with their result they considered two kinds of vector variational-like inequalities for set-valued mappings under certain new pseudomonotonicity and hemicontinuity conditions, respectively, different from the conditions that in $[12,15,18]$. In 2004, Khan at al. [9] also provided a vector version of Minty's lemma and studied two new kinds of vector variational-like inequalities by the similar method of Lee at al. [16].

Inspired and motivated by the above research work, in this paper, a more general vector version of Minty's lemma is obtained and two kinds of vector variational-like inequalities for set-valued mappings which are extensions of the corresponding vector variational-like inequalities in $[9,16]$ are considered. We show the existence of solutions to a kind of vector variational-like inequalities with set-valued mappings under certain pseudomonotonicity condition. By using the vector version of Minty's lemma and the vector variationallike inequality, we prove the existence of solutions for another type of vector variational-like inequalities with compact-valued set-valued mappings under certain hemicontinuity condition. The results presented in this paper extend and unify corresponding results of $[9,16]$.

\section{Preliminaries}

In this section, let's recall the following definitions and lemmas.

Definition 2.1 Let $D$ be a subset of a topological vector space $X$. Then a set-valued mapping $F: D \rightarrow 2^{X}$ is called KKM -mapping if for each nonempty finite subset $N$ of $D, \operatorname{CoN} \subset F(N)$, where Co denotes the convex hull and $F(N)=\bigcup\{F(u): u \in N\}$.

Definition 2.2 Let $X, Y$ be two Banach spaces and $L(X, Y)$ be a space of all linear and continuous operators of $X$ into $Y$. A bifunction $N(\cdot, \cdot)$ : $L(X, Y) \times L(X, Y) \rightarrow L(X, Y)$ is called continuous in the first argument if for any $u, v \in L(X, Y)$,

$$
\|N(u, \cdot)-N(v, \cdot)\| \rightarrow 0 \text { as }\|u-v\| \rightarrow 0,
$$

where $\|\cdot\|$ denotes some norm in $L(X, Y)$.

In a similar way, we can define the continuity of $N$ in the second argument.

Lemma 2.1 [4] Let $D$ be an arbitrary nonempty subset of a Hausdorff topological vector space $X$. Let the set-valued mapping $F: D \rightarrow 2^{X}$ be a 
KKM-mapping such that $F(u)$ is closed for all $u \in D$ and is compact for at least one $u \in D$. Then

$$
\bigcap_{u \in D} F(u) \neq \emptyset
$$

Lemma $2.2[\mathbf{1 7}]$ Let $(X,\|\cdot\|)$ be a normed vector space and $H$ be a Hausdorff metric on the collection $C(X)$ of all closed and bounded subsets of $X$, induced by a metric $d$ in term of $d(u, v)=\|u-v\|$, which is defined by

$$
H(A, B)=\max \left\{\sup _{u \in A} \inf _{v \in B}\|u-v\|, \sup _{v \in B} \inf _{u \in A}\|u-v\|\right\},
$$

for $A$ and $B$ in $C(X)$. If $A$ and $B$ are compact sets in $X$, then for each $u \in A$, there exists $v \in B$ such that

$$
\|u-v\| \leq H(A, B)
$$

Lemma 2.3 (Minty's lemma) Let $X$ be a reflexive real Banach space, $D$ a nonempty closed convex subset of $X$ and $X^{*}$ the dual of $X$. Let $T$ : $D \rightarrow X^{*}$ be a monotone and hemicontinuous operator. Then the following are equivalent:

(a) there exists an $u_{0} \in D$ such that

$$
\left\langle T\left(u_{0}\right), v-u_{0}\right\rangle \geq 0 \text { for all } v \in D
$$

(b) there exists an $u_{0} \in D$ such that

$$
\left\langle T(v), v-u_{0}\right\rangle \geq 0 \text { for all } v \in D \text {. }
$$

\section{Main results}

In this section, we state and prove the following generalized vector version Minty's lemma under the conditions different from that in $[9,16]$.

Theorem 3.1 Let $X$ and $Y$ be real Banach spaces, $D$ be a nonempty convex subset of $X$, and $\{C(u): u \in D\}$ be a family of closed convex solid cone of $Y$. Let $S, T: D \rightarrow 2^{L(X, Y)}$ be nonempty compact-valued set-valued mappings such that for any $u, v \in D, H(S(u+\lambda(v-u)), S(u)) \rightarrow 0$ and $H(T(u+\lambda(v-u)), T(u)) \rightarrow 0$ as $\lambda \rightarrow 0^{+}$, where $H$ is a Hausdorff metric defined on $L(X, Y)$ and $\eta: D \times D \rightarrow D$ an operator, suppose that the following conditions hold:

(i) $N: L(X, Y) \times L(X, Y) \rightarrow L(X, Y)$ is continuous in the first and in the second arguments, respectively;

(ii) $h: D \rightarrow Y$ is a continuous mapping ; 
(iii) $\langle N(p, q), \eta(v, v)\rangle \in C(u)$, for each $v, u \in D$ and $p \in S(v), q \in T(v)$;

(iv) the operator $u \mapsto \eta(v, u)$ of $D$ into $X$ is continuous for each $v \in D$;

(v) the operator $u \mapsto\langle N(p, q), \eta(u, v)\rangle+h(u)-h(v)$ of $D$ into $Y$ is affine for each $v \in D, p \in S(v)$ and $q \in T(v)$;

(vi) for each $u, v \in D$, there exist $s \in S(u), t \in T(u)$ such that

$$
\langle N(s, t), \eta(v, u)\rangle+h(v)-h(u) \notin-i n t C(u)
$$

implies

$$
\langle N(p, q), \eta(u, v)\rangle+h(u)-h(v) \notin \operatorname{int} C(u)
$$

for any $p \in S(v)$ and $q \in T(v)$. Then the following are equivalent:

(a) there exists an $u_{0} \in D$ such that for each $v \in D$ there exist $s \in S\left(u_{0}\right)$ and $t \in T\left(u_{0}\right)$ such that

$$
\left\langle N(s, t), \eta\left(v, u_{0}\right)\right\rangle+h(v)-h\left(u_{0}\right) \notin-i n t C\left(u_{0}\right) ;
$$

(b) there exists an $u_{0} \in D$ such that

$$
\left\langle N(p, q), \eta\left(u_{0}, v\right)\right\rangle+h\left(u_{0}\right)-h(v) \notin \operatorname{int} C\left(u_{0}\right)
$$

for all $v \in D, p \in S(v)$ and $q \in T(v)$.

Proof Suppose that there exists an $u_{0} \in D$ such that for each $v \in D$, there exist $s \in S\left(u_{0}\right), t \in T\left(u_{0}\right)$ satisfying

$$
\left\langle N(s, t), \eta\left(v, u_{0}\right)\right\rangle+h(v)-h\left(u_{0}\right) \notin-i n t C\left(u_{0}\right) .
$$

Then it follows from condition (vi) that (b) holds.

Conversely, suppose that there exists an $u_{0} \in D$ such that

$$
\left\langle N(p, q), \eta\left(u_{0}, v\right)\right\rangle+h\left(u_{0}\right)-h(v) \notin \operatorname{int} C\left(u_{0}\right) .
$$

for all $v \in D, p \in S(v)$ and $q \in T(v)$. For any arbitrary $v \in D$, letting $v_{\lambda}=\lambda v+(1-\lambda) u_{0}, 0<\lambda<1$, we have $v_{\lambda} \in D$ by the convexity of $D$. Hence for all $p_{\lambda} \in S\left(v_{\lambda}\right), q_{\lambda} \in T\left(v_{\lambda}\right)$

$$
\left\langle N\left(p_{\lambda}, q_{\lambda}\right), \eta\left(u_{0}, v_{\lambda}\right)\right\rangle+h\left(u_{0}\right)-h\left(v_{\lambda}\right) \notin \operatorname{int} C\left(u_{0}\right) .
$$

By the affinity of the operator

$$
u \mapsto\langle N(p, q), \eta(u, v)\rangle+h(u)-h(v),
$$

we have

$$
\begin{aligned}
& \left\langle N\left(p_{\lambda}, q_{\lambda}\right), \eta\left(v_{\lambda}, v_{\lambda}\right)\right\rangle+h\left(v_{\lambda}\right)-h\left(v_{\lambda}\right) \\
= & \left\langle N\left(p_{\lambda}, q_{\lambda}\right), \eta\left(\lambda v+(1-\lambda) u_{0}, v_{\lambda}\right)\right\rangle+h\left(\lambda v+(1-\lambda) u_{0}\right)-h\left(v_{\lambda}\right) \\
= & \lambda\left\langle N\left(p_{\lambda}, q_{\lambda}\right), \eta\left(v, v_{\lambda}\right)\right\rangle+\lambda h(v)-\lambda h\left(v_{\lambda}\right) \\
+ & (1-\lambda)\left\langle N\left(p_{\lambda}, q_{\lambda}\right), \eta\left(u_{0}, v_{\lambda}\right)\right\rangle+(1-\lambda) h\left(u_{0}\right)-(1-\lambda) h\left(v_{\lambda}\right) .
\end{aligned}
$$


Hence

$$
\left\langle N\left(p_{\lambda}, q_{\lambda}\right), \eta\left(v, v_{\lambda}\right)\right\rangle+h(v)-h\left(v_{\lambda}\right) \notin-i n t C\left(u_{0}\right) .
$$

In fact, suppose to the contrary that

$$
\left\langle N\left(p_{\lambda}, q_{\lambda}\right), \eta\left(v, v_{\lambda}\right)\right\rangle+h(v)-h\left(v_{\lambda}\right) \in-i n t C\left(u_{0}\right) .
$$

Since $-\operatorname{int} C\left(u_{0}\right)$ is a convex cone,

$$
\lambda\left\langle N\left(p_{\lambda}, q_{\lambda}\right), \eta\left(v, v_{\lambda}\right)\right\rangle+\lambda h(v)-\lambda h\left(v_{\lambda}\right) \in-i n t C\left(u_{0}\right) .
$$

It follows from the condition (iii) that

$$
\begin{aligned}
& (1-\lambda)\left\langle N\left(p_{\lambda}, q_{\lambda}\right), \eta\left(u_{0}, v_{\lambda}\right)\right\rangle+(1-\lambda)\left(h\left(u_{0}\right)-h\left(v_{\lambda}\right)\right) \\
= & \left\langle N\left(p_{\lambda}, q_{\lambda}\right), \eta\left(v_{\lambda}, v_{\lambda}\right)\right\rangle-\lambda\left\langle N\left(p_{\lambda}, q_{\lambda}\right), \eta\left(v, v_{\lambda}\right)\right\rangle-\lambda\left(h(v)+h\left(v_{\lambda}\right)\right) \\
\in & C\left(u_{0}\right)-\left(-\operatorname{int} C\left(u_{0}\right)\right) \\
= & \operatorname{int} C\left(u_{0}\right) .
\end{aligned}
$$

Thus

$$
\left\langle N\left(p_{\lambda}, q_{\lambda}\right), \eta\left(u_{0}, v_{\lambda}\right)\right\rangle+h\left(u_{0}\right)-h\left(v_{\lambda}\right) \in \operatorname{int} C\left(u_{0}\right),
$$

which contradicts (3.1). Hence

$$
\left\langle N\left(p_{\lambda}, q_{\lambda}\right), \eta\left(v, v_{\lambda}\right)\right\rangle+h(v)-h\left(v_{\lambda}\right) \notin-i n t C\left(u_{0}\right) .
$$

Since $S\left(v_{\lambda}\right), S\left(u_{0}\right)$ are compact, by Lemma 2.2 , for each $p_{\lambda} \in S\left(v_{\lambda}\right)$, we can find $s_{\lambda} \in S\left(u_{0}\right)$, such that

$$
\left\|p_{\lambda}-s_{\lambda}\right\| \leq H\left(S\left(v_{\lambda}\right), S\left(u_{0}\right)\right) .
$$

Since $S\left(u_{0}\right)$ is compact, without loss of generality, we may assume that $s_{\lambda} \rightarrow$ $s \in S\left(u_{0}\right)$, as $\lambda \rightarrow 0^{+}$. Moreover, we have

$$
\begin{aligned}
\left\|p_{\lambda}-s\right\| & \leq\left\|p_{\lambda}-s_{\lambda}\right\|+\left\|s_{\lambda}-s\right\| \\
& \leq H\left(S\left(v_{\lambda}\right), S\left(u_{0}\right)\|+\| s_{\lambda}-s \|,\right.
\end{aligned}
$$

since $H\left(S\left(v_{\lambda}\right), S\left(u_{0}\right)\right) \rightarrow 0$ as $\lambda \rightarrow 0^{+}$, then $p_{\lambda} \rightarrow s$ as $\lambda \rightarrow 0^{+}$. Using the same argument, we have $q_{\lambda} \rightarrow t$ as $\lambda \rightarrow 0^{+}$, where $q_{\lambda} \in T\left(v_{\lambda}\right)$ and $t \in T\left(u_{0}\right)$. By the condition (ii) and (iv), we get that $h\left(v_{\lambda}\right) \rightarrow h\left(u_{0}\right)$ and $\eta\left(v, v_{\lambda}\right) \rightarrow \eta\left(v, u_{0}\right)$ as $\lambda \rightarrow 0^{+}$.

Moreover, we have

$$
\begin{aligned}
& \left\|\left\langle N\left(p_{\lambda}, q_{\lambda}\right), \eta\left(v, v_{\lambda}\right)\right\rangle+h(v)-h\left(v_{\lambda}\right)-\left\langle N(s, t), \eta\left(v, u_{0}\right)\right\rangle-h(v)+h\left(u_{0}\right)\right\| \\
\leq & \left\|\left\langle N\left(p_{\lambda}, q_{\lambda}\right)-N(s, t), \eta\left(v, v_{\lambda}\right)\right\rangle\right\|+\left\|h\left(v_{\lambda}\right)-h\left(u_{0}\right)\right\| \\
+ & \left\|\left\langle N(s, t), \eta\left(v, v_{\lambda}\right)-\eta\left(v, u_{0}\right)\right\rangle\right\| \\
\leq & \left\|N\left(p_{\lambda}, q_{\lambda}\right)-N(s, t)\right\|\left\|\eta\left(v, v_{\lambda}\right)\right\|+\left\|h\left(v_{\lambda}\right)-h\left(u_{0}\right)\right\| \\
+ & \|N(s, t)\|\left\|\eta\left(v, v_{\lambda}\right)-\eta\left(v, u_{0}\right)\right\| \\
\leq & \left(\left\|N\left(p_{\lambda}, q_{\lambda}\right)-N\left(s, q_{\lambda}\right)\right\|+\left\|N\left(s, p_{\lambda}\right)-N(s, t)\right\|\right)\left\|\eta\left(v, v_{\lambda}\right)\right\| \\
+ & \left\|h\left(v_{\lambda}\right)-h\left(u_{0}\right)\right\|+\|N(s, t)\|\left\|\eta\left(v, v_{\lambda}\right)-\eta\left(v, u_{0}\right)\right\| .
\end{aligned}
$$


Since $\left\{\eta\left(v, v_{\lambda}\right)\right\}$ and $\left\{h\left(v_{\lambda}\right)\right\}$ are bounded and $p_{\lambda} \rightarrow s, q_{\lambda} \rightarrow t$ as $\lambda \rightarrow 0^{+}$, then

$$
\left\langle N\left(p_{\lambda}, q_{\lambda}\right), \eta\left(v, v_{\lambda}\right)\right\rangle+h(v)-h\left(v_{\lambda}\right) \rightarrow\left\langle N(s, t), \eta\left(v, u_{0}\right)\right\rangle+h(v)-h\left(u_{0}\right),
$$

as $\lambda \rightarrow 0^{+}$. It follows from $(3.2)$ and the closedness of $Y \backslash\left(-\operatorname{int} C\left(u_{0}\right)\right)$ that

$$
\left\langle N(s, t), \eta\left(v, u_{0}\right)\right\rangle+h(v)-h\left(u_{0}\right) \notin-i n t C\left(u_{0}\right)
$$

for all $v \in D$. This completes the proof.

Now we will apply our vector version of Minty's lemma (Theorem 3.1) to prove the existence of solution for a vector variational-like inequality.

Theorem 3.2 Let $X$ and $Y$ be two real Banach spaces, $D$ be a nonempty compact convex subset of $X$ and $\{C(u): u \in D\}$ be a family of closed convex solid cone of $Y$ such that for any $u \in D, C(u) \neq Y$. Let $S, T: D \rightarrow 2^{L(X, Y)}$ be set-valued mappings, $\eta: D \times D \rightarrow D$ an operator and $W: D \rightarrow 2^{Y}$ a set-valued mapping, defined by $W(u)=Y \backslash($ int $C(u))$, such that the graph $G r(W)$ is closed in $X \times Y$. Suppose that (i)-(iii), (v), (vi) hold and the following condition is satisfied:

(iv) $/$ : the operator $u \mapsto \eta(u, v)$ is continuous for each $v \in D$.

Then there exists an $u_{0} \in D$ such that $u_{0} \in D$ is a solution of the following generalized vector variational-like inequality(denoted by GVVLI-1): $\left\langle N(p, q), \eta\left(u_{0}, v\right)\right\rangle+h\left(u_{0}\right)-h(v) \notin \operatorname{int} C\left(u_{0}\right)$ for all $v \in D, p \in S(v)$ and $q \in T(v)$.

Further, if $S$ and $T$ are nonempty compact-valued set-valued mappings satisfying the condition (iv) in Theorem 3.1 and the following condition: $H(S(u+$ $\lambda(v-u)), S(u)) \rightarrow 0$ and $H(T(u+\lambda(v-u)), T(u)) \rightarrow 0$ as $\lambda \rightarrow 0^{+}$, where $H$ is a Hausdorff metric defined on $L(X, Y)$. Then there exists a $u_{0} \in D$ such that $u_{0} \in D$ is a solution of the following generalized vector variational-like inequality(denoted by GVVLI-2): find $u_{0} \in D$ such that for each $v \in D$, there exist $s \in S\left(u_{0}\right), t \in T\left(u_{0}\right)$ such that

$$
\left\langle N(s, t), \eta\left(v, u_{0}\right)\right\rangle+h(v)-h\left(u_{0}\right) \notin-i n t C\left(u_{0}\right) .
$$

Proof Define a set-valued mapping $F_{1}: D \rightarrow 2^{D}$ by

$$
\begin{aligned}
F_{1}(v)= & \{u \in D: \text { there exist } s \in S(u) \text { and } t \in T(u) \text { such that } \\
& \langle N(s, t), \eta(v, u)\rangle+h(v)-h(u) \notin-\text { int } C(u)\}
\end{aligned}
$$

for each $v \in D$. Then $F_{1}(v)$ is nonempty for each $v \in D$, since $v \in F_{1}(v)$. Note that $F_{1}$ is a KKM-mapping on $D$. In fact, suppose that $M=\left\{u_{1}, u_{2}, \cdots, u_{n}\right\} \subset$ $D, \sum_{i=1}^{n} \alpha_{i}=1, \alpha_{i} \geq 0, i=1,2, \cdots, n$ and $u=\sum_{i=1}^{n} \alpha_{i} u_{i} \notin \bigcup_{i=1}^{n} F_{1}\left(u_{i}\right)$. Then for any $s \in S(u), t \in T(u)$,

$$
\left\langle N(s, t), \eta\left(u_{i}, u\right)\right\rangle+h\left(u_{i}\right)-h(u) \in-i n t C(u)
$$


$i=1,2, \cdots, n$. Thus, we have

$$
\left\langle N(s, t), \eta\left(u_{j}, \sum_{i=1}^{n} \alpha_{i} u_{i}\right)\right\rangle+h\left(u_{j}\right)-h\left(\sum_{i=1}^{n} \alpha_{i} u_{i}\right) \in-i n t C(u)
$$

for each $j=1,2, \cdots, n$. By the affinity of the operator

$$
u \mapsto\langle N(s, t), \eta(u, v)\rangle+h(u)-h(v),
$$

it follows that

$$
\begin{aligned}
& \left\langle N(s, t), \eta\left(\sum_{j=1}^{n} \alpha_{j} u_{j}, \sum_{i=1}^{n} \alpha_{i} u_{i}\right)\right\rangle+h\left(\sum_{j=1}^{n} \alpha_{j} u_{j}\right)-h\left(\sum_{i=1}^{n} \alpha_{i} u_{i}\right) \\
= & \sum_{j=1}^{n} \alpha_{j}\left[\left\langle N(s, t), \eta\left(u_{j}, \sum_{i=1}^{n} \alpha_{i} u_{i}\right)\right\rangle+h\left(u_{j}\right)\right]-h\left(\sum_{i=1}^{n} \alpha_{i} u_{i}\right) \\
\epsilon \quad & -\operatorname{int} C(u) .
\end{aligned}
$$

By the condition (iii)

$$
\langle N(s, t), \eta(u, u)\rangle \in C(u) \cap(-\operatorname{int} C(u)),
$$

and hence $0 \in \operatorname{int} C(u)$, which contradicts $C(u) \neq Y$. Therefore, $F_{1}$ is a KKM-mapping on $D$.

Now, let us define another set-valued mapping $F_{2}: D \rightarrow 2^{D}$ by

$$
\begin{aligned}
F_{2}(v)= & \{u \in D: \forall p \in S(v), \forall q \in T(v) \text { such that } \\
& \langle N(p, q), \eta(u, v)\rangle+h(u)-h(v) \notin \text { int } C(u)\},
\end{aligned}
$$

then by the condition (vi), $F_{1}(v) \subset F_{2}(v)$ for each $v \in D$. Therefore, $F_{2}$ is also a KKM-mapping on $D$. Next, we show that for any $v \in D, F_{2}(v)$ is closed. Indeed, let $\left\{u_{n}\right\}$ be a sequence in $F_{2}(v)$ such that $u_{n} \rightarrow u_{0} \in D$, since $u_{n} \in F_{2}(v)$ for all $n, \forall p \in S(v)$ and $q \in T(v)$ such that

$$
\left\langle N(p, q), \eta\left(u_{n}, v\right)\right\rangle+h\left(u_{n}\right)-h(v) \notin \operatorname{int} C\left(u_{n}\right) .
$$

By (ii) and (iv), for all $p \in S(v), q \in T(v)$, we have

$$
\left\langle N(p, q), \eta\left(u_{n}, v\right)\right\rangle+h\left(u_{n}\right)-h(v) \rightarrow\left\langle N(p, q), \eta\left(u_{0}, v\right)\right\rangle+h\left(u_{0}\right)-h(v)
$$

as $n \rightarrow \infty$. From (3.3) and the closedness of $G r(W)$,

$$
\left\langle N(p, q), \eta\left(u_{0}, v\right)\right\rangle+h\left(u_{0}\right)-h(v) \notin \operatorname{int} C\left(u_{0}\right) .
$$

Therefore $u_{0} \in F_{2}(v)$ and so $F_{2}(v)$ is closed. Since $D$ is compact, so is $F_{2}(v)$ for all $v \in D$. Hence, by the KKM-Fan theorem

$$
\bigcap_{v \in D} F_{2}(v) \neq \emptyset
$$


then there exists an $u_{0} \in D$ such that

$$
\left\langle N(p, q), \eta\left(u_{0}, v\right)\right\rangle+h\left(u_{0}\right)-h(v) \notin \operatorname{int} C\left(u_{0}\right)
$$

for any $v \in D, p \in S(v)$ and $q \in T(v)$.

Let $S$ and $T$ are nonempty compact-valued set-valued mappings such that for any $u, v \in D, H(S(u+\lambda(v-u)), S(u)) \rightarrow 0, H(T(u+\lambda(v-u)), T(u)) \rightarrow 0$ as $\lambda \rightarrow 0^{+}$and the operator $u \mapsto \eta(v, u)$ of $D$ into $X$ is continuous for each $v \in D$, and $h: D \rightarrow Y$ be lower semicontinuous, then it follows from Theorem 3.1 that there exists an $u_{0} \in D$ such that for each $v \in D, \exists s \in S\left(u_{0}\right)$ and $t \in T\left(u_{0}\right)$ such that

$$
\left\langle N(s, t), \eta\left(v, u_{0}\right)\right\rangle+h(v)-h\left(u_{0}\right) \notin-i n t C\left(u_{0}\right) .
$$

This completes the proof of Theorem 3.2.

Remark 3.1 From Theorem 3.1, it is easy to see that the set of solutions for the GVVLI-1 is $\bigcap_{v \in D} F_{2}(v)=\bigcap_{v \in D} F_{1}(v)$, which is nonempty closed and compact, so is that for the GVVLI-2.

Remark 3.2 Theorems 3.1 and 3.2 generalize the corresponding results in $[9,16]$.

In the following, we give another existence of solutions to GVVLI-1 and GVVLI-2 without the compactness of $D$.

Theorem 3.3 Let $X, Y, C$ and $W$ be as in Theorem 3.2 and let $D$ be a nonempty, closed and convex subset of $X$. Let $N: L(X, Y) \times L(X, Y) \rightarrow$ $L(X, Y), S, T: D \rightarrow 2^{L(X, Y)}, \eta: D \times D \rightarrow D$ and $h: D \rightarrow Y$. Assume that conditions (i)-(iii), (iv), , (v), (vi) hold and the following coercive condition on $D$ is satisfied:

(vii) there exists a compact subset $K$ of $D$ and $u \in D \backslash K$ such that $\exists s \in$ $S(u), t \in T(u)$ and

$$
\langle N(s, t), \eta(v, u)\rangle+h(v)-h(u) \in-\operatorname{int} C(u),
$$

for all $v \in K$. Then the GVVLI-1 has a solution.

Further, if $S$ and $T$ are nonempty compact-valued set-valued mappings satisfying the condition (iv) and the following conditions: $H(S(u+\lambda(v-$ $u)$ ), $S(u)) \rightarrow 0$ and $H(T(u+\lambda(v-u)), T(u)) \rightarrow 0$ as $\lambda \rightarrow 0^{+}$, where $H$ is a Hausdorff metric defined on $L(X, Y)$. Then there exists a $u_{0} \in D$, such that $u_{0}$ is a solution of the GVVLI- 2 .

Proof. Let

$$
\begin{aligned}
G_{1}(v)= & \{u \in K: \exists s \in S(u), t \in T(u) \text { such that } \\
& \langle N(s, t), \eta(v, u)\rangle+h(v)-h(u) \notin-\text { int } C(u)\},
\end{aligned}
$$




$$
\begin{aligned}
G_{2}(v)= & \{u \in K: \forall p \in S(v), \forall q \in T(v) \text { such that } \\
& \langle N(p, q), \eta(u, v)\rangle+h(u)-h(v) \notin \text { int } C(u)\},
\end{aligned}
$$

for each $v \in D$. Now we shall prove $G_{1}(v) \neq \emptyset$ for each $v \in D$. It follows from condition (iii) that $v \in G_{1}(v) \neq \emptyset$ for each $v \in K$. On the other hand, for each fixed $z \in D \backslash K$, let $D_{z}=\overline{C o}\{K \cup\{z\}\}$, where $\overline{C_{o}}$ denotes the closed convex hull of a set. Since $K$ is compact, we know that $D_{z}$ is also compact. For each $v \in$ $D_{z}$, let $G(v)=\left\{u \in D_{z}: \exists s \in S(u), t \in T(u)\right.$ such that $\langle N(s, t), \eta(v, u)\rangle+$ $h(v)-h(u) \notin-$ int $C(u)\}$. It follows from the proof in Theorem 2.1, we know that there exists $u_{1} \in D_{z}$ and $s_{1} \in S\left(u_{1}\right), t_{1} \in T\left(u_{1}\right)$ such that

$$
\left\langle N\left(s_{1}, t_{1}\right), \eta\left(v, u_{1}\right)\right\rangle+h(v)-h\left(u_{1}\right) \notin-i n t C\left(u_{1}\right)
$$

for all $v \in D_{z}$. Moreover, we assert that $u_{1} \in K$. In fact, if $u_{1} \in D_{z} \backslash K \subseteq D \backslash K$, it follows from (vii) that $\exists s_{1} \in S(u), t_{1} \in T(u)$ and

$$
\left\langle N\left(s_{1}, t_{1}\right), \eta\left(v, u_{1}\right)\right\rangle+h(v)-h\left(u_{1}\right) \in-i n t C\left(u_{1}\right),
$$

which contradicts (3.5). So $u_{1} \in K$ and this implies $G_{1}(v) \neq \emptyset$ for each $v \in D_{z}$. Especially, $G_{1}(z) \neq \emptyset$. Since $z \in D \backslash K$ is arbitrary, we have $G_{1}(v) \neq \emptyset$, for all $v \in D \backslash K$. From the proof in Theorem 3.1, we can obtain that $G_{1}(v)$ is a KKM-mapping and $G_{1}(v) \subseteq G_{2}(v)$ for all $v \in D$ in view of condition (vi). We also know $G_{2}(v)$ is closed for all $v \in D$. In order to obtain our result, we only need to prove that there exists an $v_{0} \in D$ such that $G_{2}\left(v_{0}\right)$ is compact. Since $K$ is compact, $G_{2}\left(v_{0}\right) \subseteq K$. Then, $G_{2}\left(v_{0}\right)$ is also compact. The rest of the proof is the same as that in Theorem 3.2, therefore is omitted. This completes the proof.

\section{References}

1. F. Giannessi, Theorems of alternative,quadratic programmes and complementarity problems, in: R. W. Cottle, F. Giannessi, J. L. Lions (Eds.), Variational Inequalities and Complementarity Problems, Wiley, Chichester, 1980, pp. 151186.

2. G. Y. Chen, Existence of solution for a vector variational inequality: an extension of the Hartman-Stampacchia theorem, J. Optim. Theory Appl. 74(3) (1992) 445-456.

3. G. Y. Chen, X. Q. Yang, The vector complementarity problem and its equivalence with the weak minimal element in ordered sets, J. Math. Anal. Appl. 153 (1990) 136-158.

4. K. Fan, A generalization of Tychonoff's fixed point theorem, Math. Ann. 142 (1961) 305-310.

5. C. Baiacchi, A. Capelo, Variational and Quasi-variational Inequalities, Applications to Free Boundary Problems, Wiley, New York, 1984. 
6. F. Giannessi, On connections among separation, penalization and regularization for variational inequalities with point-to-set-operators, Rend. Circ. Mat. Palermo Ser. II (Suppl. 48) (1997) 11-18.

7. F. Giannessi, On Minty variational principle in: New Trends in Mathematical Programming, Kluwer Academic, Dordrecht,1997.

8. P. Hartman, G. Stampacchia, On some nonlinear elliptic differential functional equations, Acta Math. 115 (1966) 271-310.

9. M. F. Khan, Salahuddin, On generalized vector variational-like inequalities, Nonlinear Anal. 59(2004) 879-889.

10. D. Kinderlehrer, G. Stampacchia, An Introduction to Variational Inequalities, Academic Press; New York, 1980.

11. I. V. Konnov, J. C. Yao, On the generalized vector variational inequality problem, J. Math. Anal. Appl. 206(1997) 42-58.

12. A. Kumari, R. N. Mukherjee, On some generalized multi-valued variational inequalities, Indian J. Pure Appl. Math 31(6) (2000) 667-674.

13. G. M. Lee, D.S. Kim, B.S.Lee, S. J. Cho, On vector variational inequality, Bull. Korean Math. Soc. 33(4) (1996) 553-564..

14. G. M. Lee, D. S. Kim, H. Kuk, Existence of solutions for vector optimization problems, J. Math. Anal. Appl. 220(1998) 90-98.

15. B. S. Lee, G. M. Lee, D. S. Kim, Generalized vector variational-like inequalities on locally convex Hausdorff topological vector spaces, Indian J. Pure Appl. Math. 28(1) (1997) 33-41.

16. B. S. Lee, G. M. Lee, A vector version of Minty's lemma and application, Appl. Math. Lett. 12 (1999) 43-50.

17. S. B. Nadler Jr., Multi-valued contraction mappings, Pacific J. Math. 30 (1969) 475-488.

18. A. H. Siddiqi, M. F.Khan, Salahuddin, On vector variational-like inequalities, Far East J. Math. Sci. Special III (1998) 319-329.

19. S. J. Yu, J. C. Yao, On vector variational inequalities, J. Optim. Theory Appl. 89(1996) 749-769.

\section{Received: March 14, 2007}

